\title{
Tensile Crazing and Thermal History of Polyphenylquinoxaline Films
}

\author{
Wanjun LIU, Jingshu Shen, ${ }^{\dagger}$ Fengcai Lu, and Mao XU \\ Polymer Physics Laboratory, Institute of Chemistry, Chinese Academy of Sciences, \\ Beijing, 100080, People's Republic of China
}

(Received November 24, 1998)

\begin{abstract}
The effects of thermal history on the tensile crazing of polyphenylquinoxaline (PPQ-E) films were studied. Crazing of PPQ-E films during stretching was in situ observed by SEM. Critical strain for craze initiation and craze stability depended on thermal history of the samples. The DSC endothermic peak at the glass transition region of the samples with different pre-heating conditions was interpreted in terms of the cohesional entanglement theory. KEY WORDS Polyphenylquinoxaline / Crazing / Thermal History / Cohesional Entanglement /

Scanning Electron Microscope / Differential Scanning Calorimetry /
\end{abstract}

Crazing is characteristic of polymers. Failure of polymers is often preceded by crazing. Especially when a glassy polymer is stretched, craze can be found on the surface and in the interior of the materials. Subsequently, craze will develop into crack and lead to fracture of the polymer material. ${ }^{1}$ In addition, structure recovery in polymer is a process from thermodynamic nonequilibrium state to true equilibrium state. ${ }^{2}$ Under the recovery process enthalpy and volume change. This phenomenon is called physical aging. ${ }^{3,4}$ Associated with the physical aging mechanical properties will change. ${ }^{5-8} \mathrm{~A}$ few studies have focused on the effects of physical aging on polymer crazing behavior.

Shen et al. studied the effects of physical aging on crazing for poly(methyl methacrylate) (PMMA) films. ${ }^{9}$ Arnold ${ }^{10}$ demonstrated that a longer physical aging time delayed craze formation in polystyrene (PS), but once formed, crazes were much less stable than those formed in a short aging time. Gloria et al. ${ }^{11}$ found that the critical crazing strain of PS decreased with increasing aging temperature and aging time. However, Plummer and Donald $^{12}$ found that strain to crazing is constant for different aging times.

Polyphenylquinoxaline (PPQ-E) is an aromatic heterocyclic polymer with high performance and high tempera-

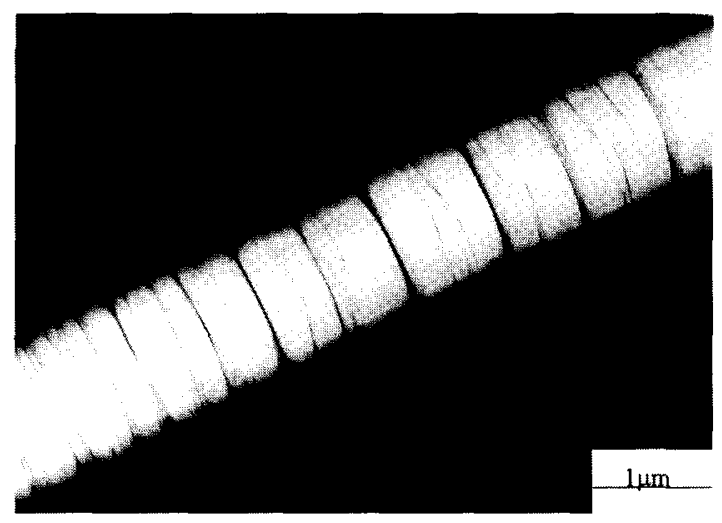

Figure 1. Transmission electron micrograph of example of craze in PPQ-E.

${ }^{\dagger}$ To whom correspondence should be addressed. ture resistance and water resistance. It is mainly used to coat wire in submerged electric motors. It can form PPQE-metallic complexes with high catalytic activity and be transformed into conductor, and pervaporation and UF membranes. ${ }^{13-16}$ PPQ-E under tensile stress or axially applied stress shows crazing in a previous study. ${ }^{17}$ An example of TEM micrograph of craze is shown in Figure 1. This manifests that craze of PPQ-E consists of fibrils and microvoids and there is obvious interface between craze and bulk.

In this paper, correlation between thermal history and crazing was sought under two pre-heating conditions for PPQ-E, quenching from temperature above $T_{\mathrm{g}}$ and slowly cooling from temperature above $T_{\mathrm{g}}$. Crazing, craze morphology, critical strain values for craze initiation and craze stability of PPQ-E films with the two different states were studied. Crazing was in situ observed by SEM during stretching process.

\section{EXPERIMENTAL}

PPQ-E was synthesized from aromatic tetraamine and aromatic tetraketone by condensation polymerization as shown in eq $1 .^{13,16}$ Monomers of $3,3^{\prime}, 4,4^{\prime}$-tetraamiodiphenyl ether and 1,4-bis(phenylglyoxalyl)benzene were prepared according to the literature. ${ }^{14,18}$

PPQ-E synthesized in the laboratory was cast into a film $0.07 \mathrm{~mm}$ in thickness. The film was cut to obtain dumbbell specimens in which the parallel part was approximately $4.8 \mathrm{~mm}$ in length and $2.2 \mathrm{~mm}$ in width. The specimens were annealed at $320^{\circ} \mathrm{C}$ for $1.5 \mathrm{~h}$ in nitrogen gas to eliminate past mechanical history. They were subjected to two thermal histories, slowly cooling to room temperature (denoted as sample 1) and quenching to

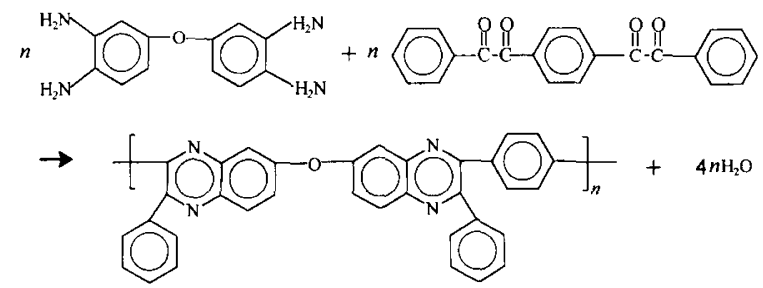

Equation 1. Synthesis of polyphenylquinoxaline. 


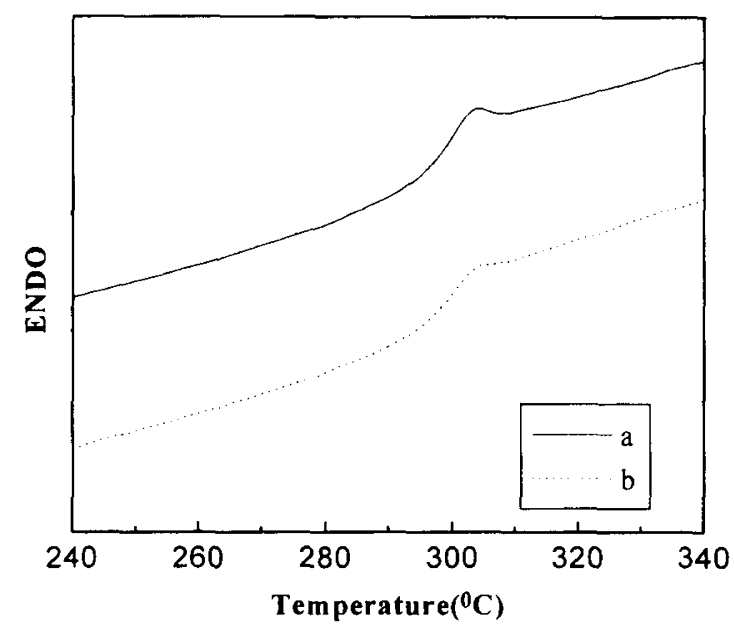

Figure 2. DSC curves for (a) sample 1 and (b) sample 2 .

room temperature (denoted as sample 2). Thus, two groups of samples with different thermal histories were obtained.

The crazing of samples was in situ observed during uniaxial stretching at a strain rate of $2 \% \mathrm{~min}^{-1}$ by Hitachi S-570 SEM. The displacement-load curve during stretching was simultaneously measured. Samples were coated with a thin layer of gold prior to SEM observation. Micrographs of the crazing were obtained by camera. Critical strain for craze initiation was determined by observation in the tensile process, not by micrographs after load relaxation.

The glass transition temperature $\left(T_{g}\right)$ and endothermic peak at the glass transition region were measured using TA 2100 Modulated DSC with heating rate 10 ${ }^{\circ} \mathrm{C}$ min $^{-1}$. The area of the endothermic peak was obtained through integration (denoted as, $\Delta H$ ).

\section{RESULTS AND DISCUSSION}

DSC curves of samples with two different thermalhistories are shown in Figure 2. Curve (a), slowly cooling sample DSC curve, shows an obvious endothermic peak at glass transition region. But the endothermic peak of curve (b) for quenching sample is not obvious. All the results are related to thermal history of samples. Qian et $a l$. introduced a new point of view for entanglement ${ }^{19,20}$ on the basis of more experiments. This theory assumes that in addition to topological entanglement of chains, there is cohesional entanglement which is the site of local nematic interaction between segments of a few monomer units or three nearest neighboring chains serving as the physical crosslinks to render the linear polymer to behave as a network. The density of cohesional entanglements is more than that of topological entanglements. Because cohesional entanglement is a weak interaction, the density of the cohesional entanglement points is strongly dependent on temperature. Therefore, the strength and quantity of entanglement are related to the thermal history of samples. The cohesional entanglement is important for physical properties of a polymer around and below its $T_{\mathrm{g}}$. According to this point of views, for the sample annealed above $T_{\mathrm{g}}$ temperature and quenched to room temperature, molecular
Table I. Areas of endothermic peaks $(\Delta H)$ for two samples

\begin{tabular}{ccc}
\hline Sample & 1 & 2 \\
\hline$\Delta H / \mathrm{J} \mathrm{g}^{-1}$ & 1.12 & 0.08 \\
\hline
\end{tabular}

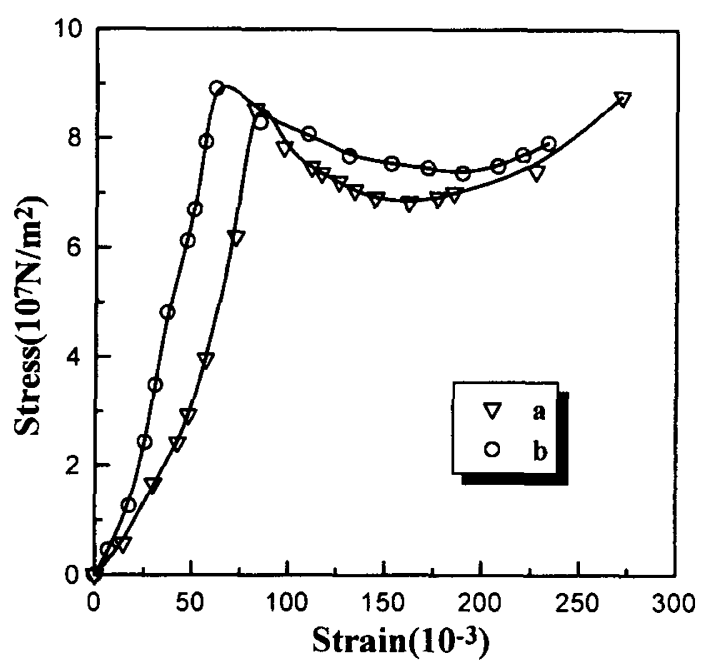

Figure 3. Nominal stress-strain curves for (a) sample 1 and (b) sample 2 .

chains in the sample are frozen at the state above $T_{\mathrm{g}}$. Thus, molecular chains have no chance to rearrange, and quenched samples should be in thermodynamic nonequilibrium in which the state of cohesional entanglement remains the same above $T_{\mathrm{g}}$. But for the slowly cooling samples, slowly cooling is in fact relaxation of molecular chains. This makes molecules rearrange and form more and stronger cohesional entanglement points among the molecules. Therefore, in the heating of DSC, sample 1 absorbs more energy to disentangle and obvious endothermic peak may appear at the glass transition region. The areas of endothermic peak on DSC curves for two samples are shown in Table I. The differences in the areas of two endothermic peaks reflect the disparity of cohesional entanglements between sample 1 and sample 2 . This result is similar with that of Richardon and Savil. ${ }^{21}$

For slowly cooling samples, the nominal stress-strain curve is shown in Figure 3a. When the strain approached $7.24 \%$, a few minute crazes having obvious fiberized character began to appear as shown in Figures $4 \mathrm{a}$ and $4 \mathrm{~b}$. After the yielding point at strain of $11.11 \%$, much craze was generated and developed within the whole view. Most craze is separated and only a few adjacent crazes are connected. With further stretching, craze propagates. As the strain reached $15.56 \%$, the crazing developed over the whole sample, Figure 4c. Some of craze ends by the scratch on the surface and others changed growth direction due to the scratch. With higher magnification some crazes linked together and fibrils fractured, while other smaller crazes did not link. At strain of $22.22 \%$, as shown in Figure $4 \mathrm{~d}$, most craze progressed into microcracks. The sample was broken at strain of $26.22 \%$.

The quenching sample underwent annealing above $T_{\mathrm{g}}$ and quenched to room temperature. Its nominal stress ${ }^{-}$ strain curve is shown in Figure 2b. When strain reached 


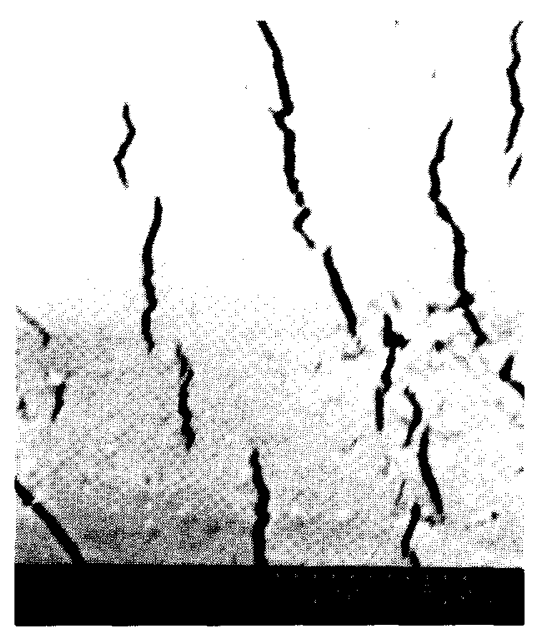

[a]

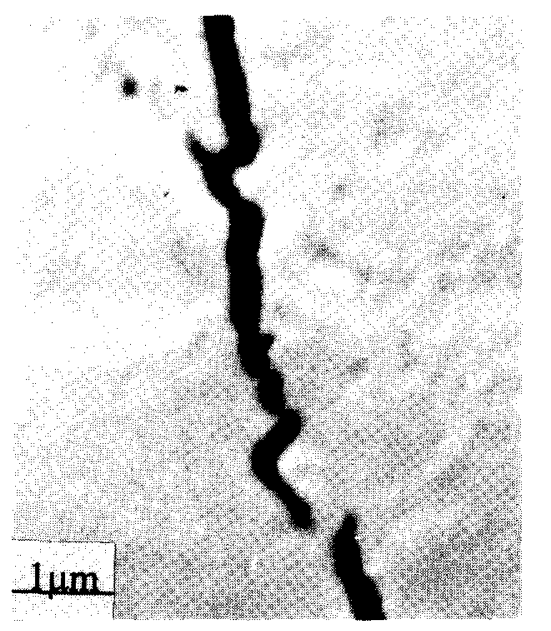

[b]

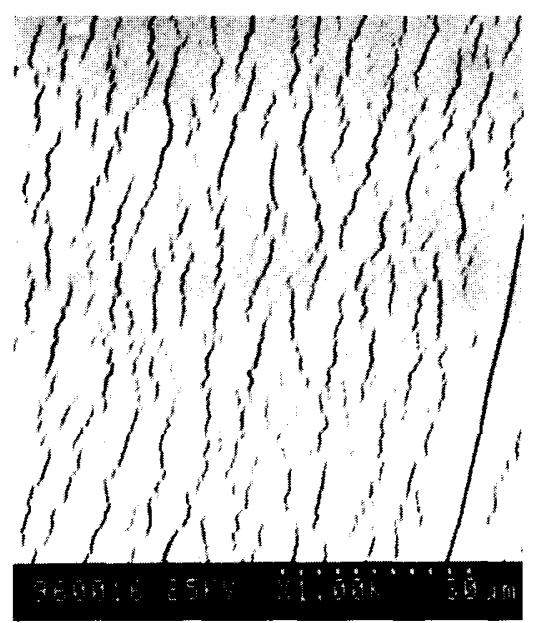

[c]

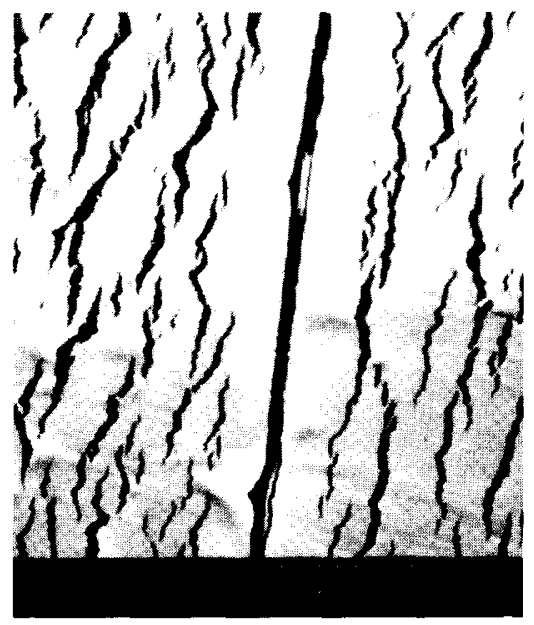

[d]

Figure 4. Scanning electron micrographs of crazing in sample 1 at different strain values (a) $7.24 \%$, (b) $7.24 \%$, (c) $15.56 \%$ and (d) $22.22 \%$.

$3.72 \%$, minority of slim and short craze began to appear as shown in Figures $5 \mathrm{a}$ and $5 \mathrm{~b}$. Crazing propagated slowly with more stretching. Upon reaching a strain of $9.24 \%$ after the yielding point, crazes filled up whole view (Figure $5 \mathrm{c}$ ). These crazes are homogeneous in size and arranged regularly. Crazing developed rapidly under further loading. At strain of $18.76 \%$, as shown in Figure $5 \mathrm{~d}$, crazes become thicker or wider and some microvoid or microcracks appear. As strain reaching $23.30 \%$, this material was fractured.

In the above experiments the critical strain for craze initiation, $\varepsilon_{\mathrm{c}}$, and strain at fracture, $\varepsilon_{\mathrm{b}}$, of the samples can be determined. $\left(\varepsilon_{\mathrm{b}}-\varepsilon_{\mathrm{c}}\right)$ is used to characterize craze stability. The values of $\varepsilon_{\mathrm{c}}$ and $\left(\varepsilon_{\mathrm{b}}-\varepsilon_{\mathrm{c}}\right)$ are summarized in Table II. It was found that $\varepsilon_{\mathrm{c}}$ and $\left(\varepsilon_{\mathrm{b}}-\varepsilon_{\mathrm{c}}\right)$ are dependent on thermal-dealing of samples.

$\varepsilon_{\mathrm{c}}$ of sample 1 is higher than that of sample 2 . The craze stability of sample 1 is lower than that of sample 2 . Thus, for slowly cooled sample the critical crazing appears later, craze initiation is difficult and craze is less stable. Crazing of quenched sample appears earlier, craze forms easily and also craze is more stable. Actually, quickly cooling or slowly cooling is analogous to quenching or physical aging respectively. In other words, cooling rate after annealing above $T_{g}$ has influence on craze formation. These results are consistent with that of Arnold. ${ }^{10}$

Samples 1 and 2 have the same molecular weight. Under the same strain rate, the effects of topological entanglement on sample 1 and sample 2 are identical. According to the point of view of cohesional entanglements, ${ }^{19}$ for the two samples, the main difference in condensed state is the quantity and strength of cohesional entanglements in the samples. More cohesional entanglement points formed in sample 1 than in sample 2, molecular motion was more constrained and the release of cohesional entanglements points should absorb more energy. Thus, initial crazing should occur at higher stretching strain. Therefore, the critical strain for craze initiation is related to pre-heating, i.e., cohesional entanglements in PPQ-E.

In consequence of entanglements of polymer chains an entangled network is formed. Michler suggests that the molecular mobility of local areas increases during locally stretching the network and assumes that the meshes of the entangled network contain many other molecular segments only weakly bonded and relatively loosely packed. ${ }^{22}$ The material in mesh is more easily deformable than the surrounding material. Hence some small elongated flow domain arise under tensile stress. These domains that act as craze nuclei lead to craze initiation. The flow domain is limited by the neibouring more 


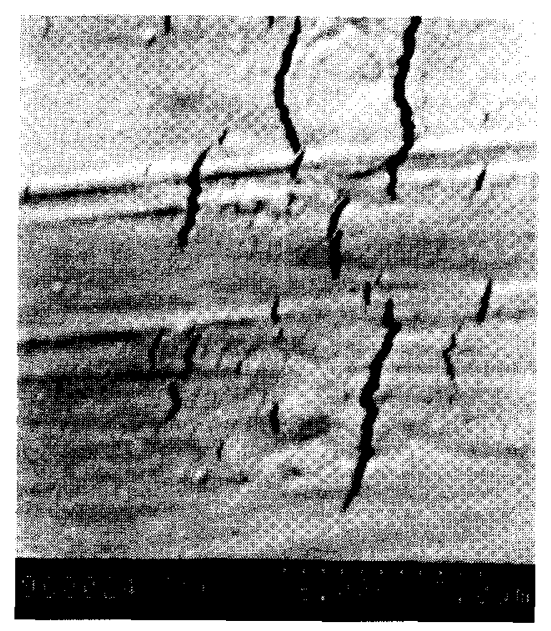

[a]

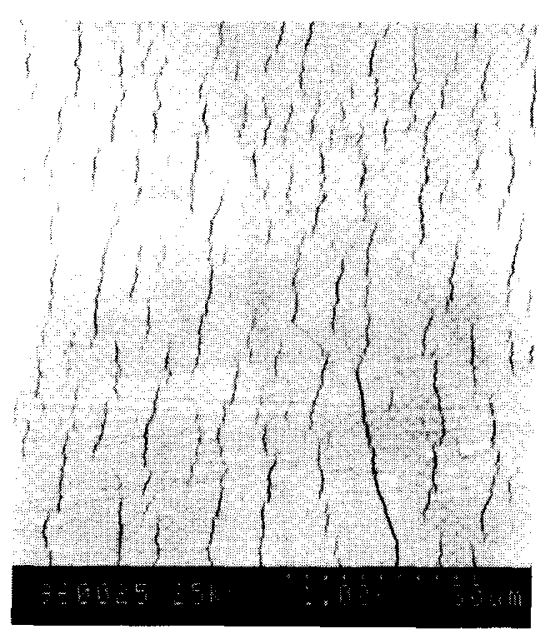

[c]

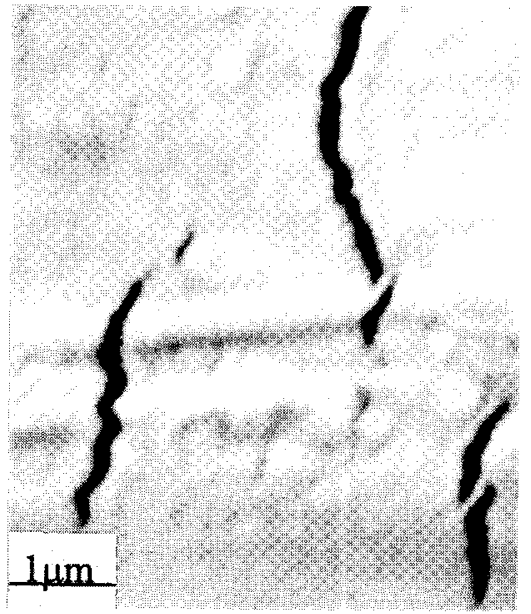

[b]

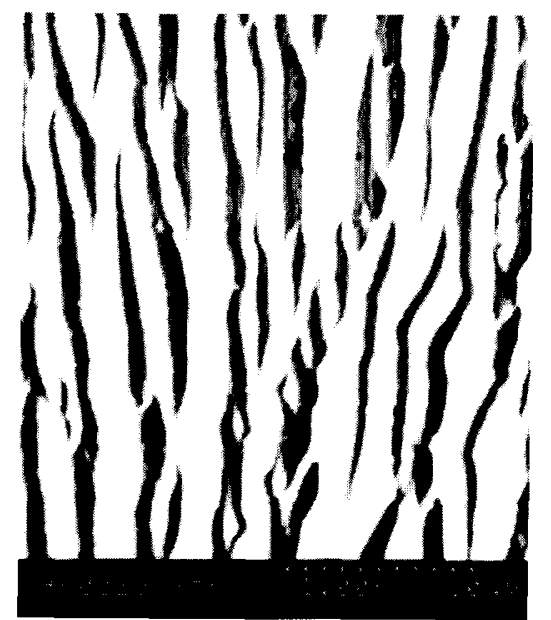

[d]

Figure 5. Scanning electron micrographs of crazing in sample 2 at different strain values (a) $3.72 \%$, (b) $3.72 \%$, (c) $9.24 \%$ and (d) $18.76 \%$.

Table II. Critical strain for craze initiation and stability of two samples

\begin{tabular}{lrr}
\hline Sample & 1 & 2 \\
\hline$\varepsilon_{\mathrm{c}} / \%$ & 7.24 & 3.72 \\
$\varepsilon_{\mathrm{b}}-\varepsilon_{\mathrm{c}} / \%$ & 18.98 & 19.60 \\
\hline
\end{tabular}

strongly entangled chains. The density of flow domain depends on the entanglement density and degree of activity of the domains. The effect of topological entanglement on crazing is constitutive, namely, topological entanglement of polymer decide deformation mechanism of the polymer, crazing or shear flow. ${ }^{23-25}$ In Michler's model, ${ }^{22}$ the activity of the domains is controlled by thermal history of the polymer. Actually, thermal treatment changes the state of cohesional entanglements among polymer chains. In this case, it is reasonable to consider the density of flow domain depends on density and strength of the cohesional entanglement point. In our experiments, samples have identical molecular weight and the effects of topological entanglement on crazing are identical. Cooling rate after thermal dealing above $T_{\mathrm{g}}$ should affect the density and strength of cohesional entanglement points, thus, influence the density of flow domain, and cause craze initiation. To initiate craze during stretching, opening entanglement points, decreasing co- hesional entanglement strength and density and increasing the degree of activity of the domains are necessary. Thereby, for slowly cooled sample, craze initiation requires more energy and crazing occurs at higher critical strain values.

Acknowledgments. This work was supported by the National Key Projects for Fundamental Research"Macromolecular Condensed State", the State Science and Technology Commission of China and by the Science Foundation of PPLAS.

\section{REFERENCES}

1. R. W. Cahn, P. Haasen, and E. J. Krammer, "Materials Science and Technology", Vol. 12, VCH(Verlagsgesellschaft $\mathrm{mbH}$ ), London/Weinheim, 1993, Chapter 15, p 699.

2. J. M. Hutchinson, Prog. Polym. Sci., 20, 703 (1995).

3. J. Perez, J. Y. Cavaille, R. D. Calleja, L. G. Ribbles, M. M. Pradas, and A. R. Greus, Makromol. Chem., 192, 2141 (1991).

4. L. C. E. Struik, Polymer, 28, 1869 (1987).

5. L. J. Broutman and S. M. Krishnakumar, Polym. Eng. Sci., 16, 74 (1976).

6. J. M. Crissman and G. B. Mckenna, J. Polym. Sci. Polym. Phys. Ed., 25, 1167 (1987).

7. A. J. Hill, K. J. Heater, and C. M. Arawal, J. Polym. Sci. Polym. Phys. Ed., 28, 387 (1990).

8. C. Bauwent-crowet and J. C. Bauwens, Polymer, 23, 1599 
(1982).

9. W. Ye and J. Shen, Acta Polymerica Sinica (Chinese), 3, 380 (1995).

10. J. C. Arnold, Polym. Eng. Sci., 35, 165 (1995).

11. G. M. Guster and G. B. Mckenna, Polym. Prepr., Am. Chem. Soc., Div. Polym. Chem., 36, 63 (1995).

12. C. J. G. Plummer and A. M. Donald, J. Polym. Sci., Polym. Phys. Ed., 27, 325 (1989).

13. F. Lu, X. Sui, B. Wang, J. Chang, L. Xing, Y. Wang, Y. Gao, and L. Bai, Polym. Commun. (Chinese), 3, 146 (1980).

14. L. Xing, L. Bai, and F. Lu, Acta Polymerica Sinica (Chinese), 4, 478 (1992)

15. M. Piao, M. Wan, and F. Lu, J. Appl. Polym. Sci., 69, 123 (1998).

16. F. Lu, J. Macromol. Sci.-Rev. Macromol. Chem. Phys., C38,
143 (1998).

17. W. Liu, J. Shen, F. Lu, and M. Xu, unpublished.

18. R. J. Foster and C. S. Marvel, J. Polym. Sci., A3, 417 (1965)

19. R. Qian, Macromol. Symp., 124, 15 (1997).

20. R. Qian, L. Wu, D. Shen, H. N. Donald, A. M. Richard, and F. S. David, Macromolecules, 26, 2950 (1993)

21. M. J. Richardson and N. G. Savil, Polymer, 18, 413 (1977).

22. G. H. Michler, Makromol. Chem., Macromol. Symp., 41, 39 (1991).

23. A. M. Donald and E. J. Kramer, Polymer, 23, 1183 (1982).

24. A. M. Donald and E. J. Kramer, J. Mater. Sci., 17, 1781 (1982).

25. A. M. Donald and E. J. Kramer, J. Polym. Sci. Polym. Phys. Ed., 20, 899 (1982). 